\title{
Study of the Behavior of a Bell-Shaped Colonic Self-Expandable NiTi Stent under Peristaltic Movements
}

\author{
Sergio Puértolas, ${ }^{1}$ Eduardo Bajador, ${ }^{2}$ José A. Puértolas, ${ }^{3}$ Enrique López, ${ }^{4}$ Elena Ibarz, ${ }^{1}$ \\ Luis Gracia, ${ }^{1}$ and Antonio Herrera ${ }^{5,6}$
}

${ }^{1}$ Department of Mechanical Engineering, University of Zaragoza, Maria de Luna 3, 50018 Zaragoza, Spain

${ }^{2}$ Department of Gastroenterology, Miguel Servet University Hospital, Paseo Isabel la Católica 1, 50009 Zaragoza, Spain

${ }^{3}$ Department of Material Science, University of Zaragoza, Maria de Luna 3, 50018 Zaragoza, Spain

${ }^{4}$ Department of Manufacturing Engineering, University of Zaragoza, Maria de Luna 3, 50018 Zaragoza, Spain

${ }^{5}$ Department of Surgery, University of Zaragoza, Domingo Miral, 50009 Zaragoza, Spain

${ }^{6}$ Department of Orthopaedic Surgery and Traumatology, Miguel Servet University Hospital, Paseo Isabel la Católica 1, 50009 Zaragoza, Spain

Correspondence should be addressed to Luis Gracia; lugravi@unizar.es

Received 23 January 2013; Revised 6 May 2013; Accepted 21 May 2013

Academic Editor: Xin-yuan Guan

Copyright (C) 2013 Sergio Puértolas et al. This is an open access article distributed under the Creative Commons Attribution License, which permits unrestricted use, distribution, and reproduction in any medium, provided the original work is properly cited.

\begin{abstract}
Managing bowel obstruction produced by colon cancer requires an emergency intervention to patients usually in poor conditions, and it requires creating an intestinal stoma in most cases. Regardless of that the tumor may be resectable, a two-stage surgery is mandatory. To avoid these disadvantages, endoscopic placement of self-expanding stents has been introduced more than 10 years ago, as an alternative to relieve colonic obstruction. It can be used as a bridge to elective single-stage surgery avoiding a stoma or as a definitive palliative solution in patients with irresectable tumor or poor estimated survival. Stents must be capable of exerting an adequate radial pressure on the stenosed wall, keeping in mind that stent must not move or be crushed, guaranteeing an adequate lumen when affected by peristaltic waves. A finite element simulation of bell-shaped nitinol stent functionality has been done. Catheter introduction, releasing at position, and the effect of peristaltic wave were simulated. To check the reliability of the simulation, a clinical experimentation with porcine specimens was carried out. The stent presented a good deployment and flexibility. Stent behavior was excellent, expanding from the very narrow lumen corresponding to the maximum peristaltic pressure to the complete recovery of operative lumen when the pressure disappears.
\end{abstract}

\section{Introduction}

Colorectal cancer is the second most prevalent cancer in the world with incidence of one million new cases per year and mortality of about 529,000 deaths [1]. In advanced stages, the tumor tends to grow inside the colon lumen, causing stenosis that may block the passage of stool. Obstruction has been reported in 7-29\% of patients with colorectal cancer [2]. Patients with malignant large-bowel obstruction tend to have advanced disease and be poor surgical candidates.

The traditional method of managing complete or subtotal cancer colonic obstruction is surgical, but in the emergency setting, surgery carries a high mortality (15-20\%) and high morbidity (45-50\%) with increased prevalence of intensive care stay, infections, and complications related to stomas [3].

Moreover, even when the tumor is surgically resectable, after the tumor excision, the surgeon should make a temporary colostomy, because a dirty colon cannot be shunted. Therefore, the initial surgery (resection of the primary tumor and colostomy) must be followed by a second intervention to perform the intestinal anastomosis sometime after.

To avoid these disadvantages, endoscopic placement of self-expanding metal stents (SEMS) to relieve colonic obstruction has been introduced more than 10 years ago [4]. 


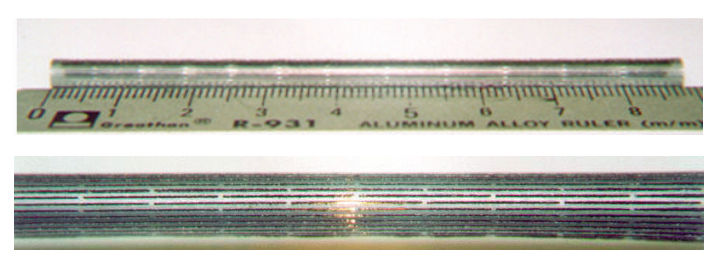

(a)

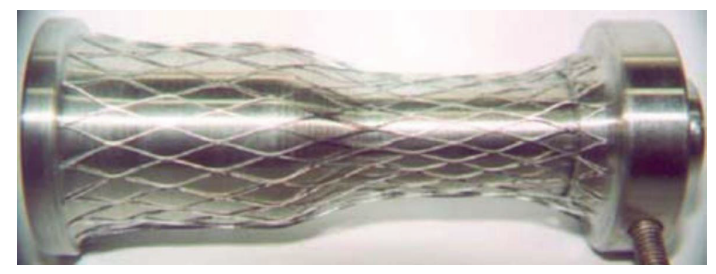

(b)

FIGURE 1: Stent manufacturing: (a) slotted tube, (b) stent after expansion process.

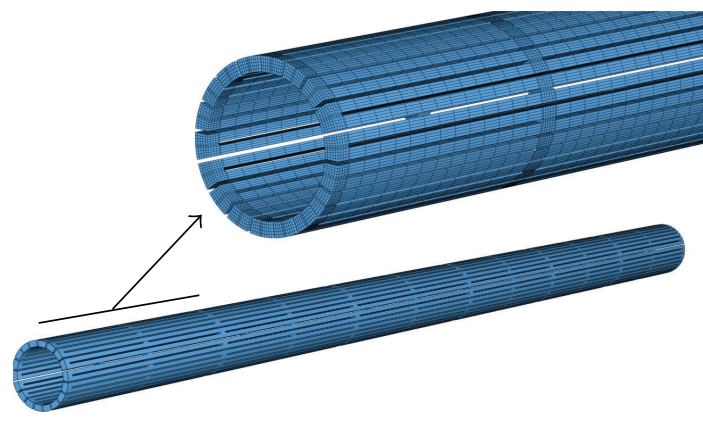

(a)

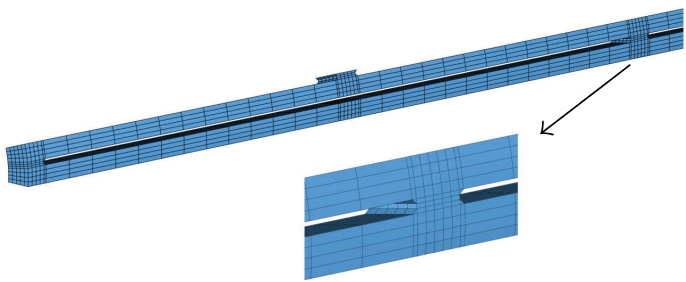

(b)

FIgUrE 2: FE model of the slotted tube: (a) global model, (b) detail of knot between struts.

If the patient is a poor candidate for surgical resection because of underlying illness or has unresectable or very widespread metastasis, stenting is a widely accepted alternative to surgical intervention (i.e., stenting for palliation). When used for this purpose, an important factor to consider is the duration of stent patency. In a recent multicenter study, there is a stent patency of $96 \%$ [5], on the other hand if we compare the results of treatment of obstruction by colostomy or SEMS, these have fewer complications, are less costly, and have shorter hospital stay [6]. If the patient has a surgical resectable tumor, the SEMS is used as "bridge" to elective single-stage surgery, most often without a stoma, thereby significantly reducing the mortality and morbidity [7-10].

Recent technologic advances have seen the advent of stents that expand to greater diameters and have increased flexibility, facilitating placement across tortuous paths and more angulated strictures, like those encountered in the colon [11-13]. Stents must be capable of exerting a radial pressure on the bowel wall stenosed, allowing bolus transit, while keeping good flexibility to adapt well to the walls and prevent migration or obstruction. The peristaltic wave which allows the intestinal transit affects the stent, which must not move or crushed, guaranteeing an adequate lumen. A specific finite element (FE) simulation concerning peristaltic movements affecting the global behavior of the stent would help to know better its response. Therefore this work tries to simulate how the forces generated by intestinal peristalsis affect the stent functionality.

\section{Materials and Methods}

The stent geometry analyzed in this paper corresponds to the prototype proposed by the same authors in $[11,14]$. It is a self-expanding stent with diamond cell type and bellshaped profile with longitudinal variable radial strength. The stent was manufactured in the laboratory of Material Science Department, under authors' supervision, from a NiTi thin-walled tube of $4.5 \mathrm{~mm}$ outer diameter with a wall thickness of $400 \mu \mathrm{m}$, with a composition of 50.8 at $\%$ nickel and 49.2 at\% titanium (supplied by Minitubes Ltd, Grenoble, France). A laser-cutting technique was used to obtain a strut width of $400 \mu \mathrm{m}$ and variable strut length from $6.15 \mathrm{~mm}$ to $16 \mathrm{~mm}$ (Figure 1(a)). The final bell-shaped stent was obtained by plastic deformation process at cryogenic conditions and posterior thermal annealing treatment $[11,14]$ (Figure 1(b)). A more detailed explanation about the whole process including thermal treatments can be found in [14] for a preliminary design. The final design reported in this work, with the desired features according clinical requirements, was achieved after several trials. That configuration is denoted as undeformed (zero load state, stress free) configuration.

In order to get an accurate geometric model, the previous deformation process was simulated, starting with the slotted tube, expanding and fixing its final shape. In this respect, a $3 \mathrm{D}$ finite element model was developed (Figure 2), from a solid 3D CAD model, using brick and wedge linear elements. The total number of elements in the model was 184770 (180450 brick type, 4320 wedge type). The forming process was simulated in the way described in [14], using a rigid surface for the forming tool inserted in the tube. This is a realistic assumption, taking into account that its stiffness is several orders of magnitude upper to stent structure. The geometric model and subsequent meshing were made using the I-Deas software [15]. Contact conditions (frictionless) were imposed between the stent-node-based surface (innernode set) and the outer surface of the forming tool, and 
a user-defined material subroutine (UMAT) for the NiTi mechanical behavior, based on the developments included in [16], was employed using Abaqus Standard 6.10.1 software [17], after previously carrying out an adjustment of parameters from the results obtained in tensile tests (Figure 3). Once the final shape was achieved (Figure 4), the stress and strain fields were removed.

Usually, the stent is mounted in a preloaded constrained position on a delivery catheter. When the obstruction has been localized, a guire is passed through the lumen beyond the stenosis and then the stent is advanced over it and positioned across the stricture. Then, the constraining mechanism is released and the expansive forces cause radial opening of the lumen. Then, in order to get a more realistic simulation, both catheter introduction and liberation were simulated in two steps.

(a) Crimping: the stent was compressed into a $14 \mathrm{Fr}$ catheter $(1 \mathrm{Fr}=0.333 \mathrm{~mm})$ defined as a rigid surface. The stent was fixed in axial direction and free on radial direction allowing deforming freely under the contact pressure of a crimping tool and catheter with no contact friction.

(b) Releasing: while holding the stent fixed in axial direction and slipping the rigid surface that models the delivery system with frictionless contact, the stent was progressively recovering its shape but limited by the compressive force exerted by the colonic tissue.

Since the colon transports solid material, it is necessary to cause its movement through a local contraction of great strength, which is spreading in waves stretches that resemble a worm motion. So, after positioning the stent, the application of a pressure wave on the external surface of the ductstent structure is modeled to simulate the response to peristaltic movement, which is the most demanding mechanical requirement. The loading conditions consider a wave motion with amplitude which produces a uniform constriction in the section of duct stent that has a diameter at maximum compression of $6 \mathrm{~mm}$, according to clinical experience. A ring compression was modeled as a displacement driven process. That option was choosen after studying different alternatives (displacement wave, pressure wave) and after verifying in clinical experimentation that the radial recovering force of the stent was appropriate when it is reached by the peristaltic wave, suffering the maximum radial compression, being able to achieve a complete recovering when the peristaltic pressure disappears.

Concerning material properties, NiTi has a super elastic behaviour which lends to the property of withstanding large elastical deformations with relatively low tensions. This property is due to the change of phase austenite-martensiteaustenite which the material undergoes when it is subjected to tension. The shape of hysteresis curve characteristic of NiTi alloys allows achieving a calibrated behavior of the stent, providing forces adapted to clinical needs to treat each stenosis.

There were several uniaxial tensile tests using a universal testing machine, Instron 5565. Each NiTi test specimen

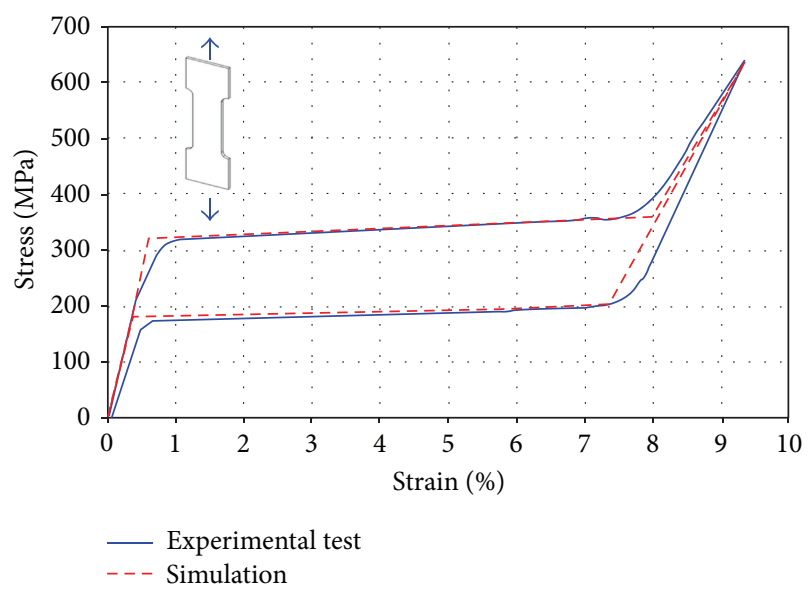

FIgURE 3: Adjustment of parameters for UMAT subroutine from the results obtained in tensile tests.

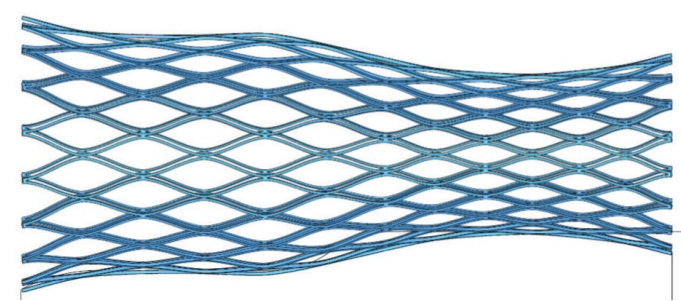

(a)

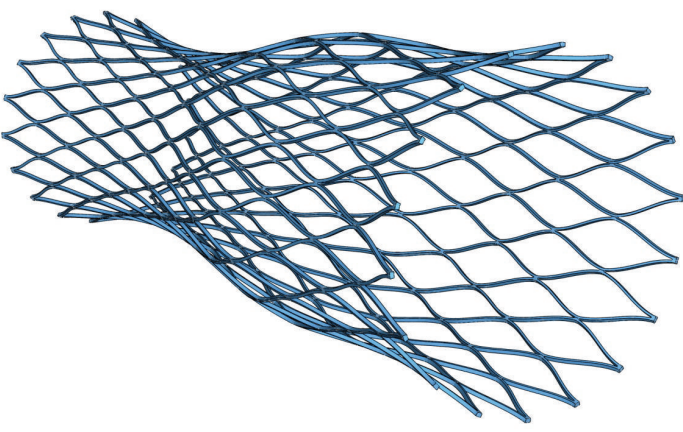

(b)

FIGURE 4: FE model of the expanded stent.

(dog-bone shaped, $60 \mathrm{~mm}$ long, $10 \mathrm{~mm}$ width, $1 \mathrm{~mm}$ thick, obtained from a sheet supplied by the same manufacturer than the tube and with the same composition and properties) was subjected to complete cycle of loading and unloading, making a displacement control. During load cycle the material reaches complete transformation to martensite, getting maximum strain of $8 \%$. Young modulus of the austenite and martensite phases $\left(E_{\mathrm{A}}, E_{\mathrm{M}}\right)$, the different values of phase transition stresses $\left(\sigma_{s}^{\mathrm{AM}}, \sigma_{f}^{\mathrm{AM}}, \sigma_{s}^{\mathrm{MA}}, \sigma_{f}^{\mathrm{MA}}\right)$ for a specific reference temperature $\left(22^{\circ} \mathrm{C}\right)$, and maximum strain $\left(\varepsilon_{L}\right)$ were derived from the experimental tests (Table 1, Figure 3).

Colonic tissue has been modeled using a homogeneus, isotropic, and hyperelastic constitutive model. The constitutive law is described in terms of a strain energy density 
TABLE 1: NiTi material parameters which define its stress-strain curve. UMAT input material parameters for constitutive model implemented in Abaqus.

\begin{tabular}{llc}
\hline Parameter & Description & Value \\
\hline$E_{\mathrm{A}}$ & Austenite elasticity modulus & $52650 \mathrm{MPa}$ \\
$\nu_{\mathrm{A}}$ & Austenite Poisson's ratio & 0.33 \\
$E_{\mathrm{M}}$ & Martensite elasticity modulus & $38250 \mathrm{MPa}$ \\
$\nu_{\mathrm{M}}$ & Martensite Poisson's ratio & 0.33 \\
$\varepsilon_{\mathrm{L}}$ & Maximum transformation strain & $8 \%$ \\
$\sigma_{s}^{\mathrm{AM}}$ & Start of transformation & $320 \mathrm{MPa}$ \\
$\sigma_{f}^{\mathrm{AM}}$ & austenite-martensite stress & \\
$\sigma_{s}^{\mathrm{MA}}$ & End of transformation & $360 \mathrm{MPa}$ \\
& austenite-martensite stress & \\
$\sigma_{f}^{\mathrm{MA}}$ & Start of transformation & $200 \mathrm{MPa}$ \\
$T_{0}$ & martensite-austenite stress & \\
$C^{\mathrm{AM}}$ & martensite-austenite stress & $180 \mathrm{MPa}$ \\
$C^{\mathrm{MA}}$ & Reference temperature & $22^{\circ} \mathrm{C}$ \\
\hline
\end{tabular}

function, taken as a polynomial Mooney-Rivlin form [19]. Only a few studies concerning mechanical behaviour of colonic tissue can be found in the specialized literature [18, 20], so in this work the data from [18] were used (Figure 5).

Finally, in order to check the reliability of the simulation, a clinical experimentation with porcine specimens was carried out. For stenosis creation, through laparotomy, an autologous peritoneal patch measuring $6 \mathrm{~cm}$ in length and $4 \mathrm{~cm}$ width was harvested and folded into a double-layer band $(6 \times$ $2 \mathrm{~cm})$. The peritoneal band was wrapped around the sigmoid colon approximately $20 \mathrm{~cm}$ from the anus. A $6 \mathrm{~mm}$ diameter probe was placed inside the colon and the band was then tightened and sutured onto the colon. Finally, a contrast enema was performed to document the presence of stenosis [14]. The colon was simulated as a hyperelastic cylindrical duct with a mesh of 18144 membrane elements with a thickness of $0.9 \mathrm{~mm}$, with a stiffness accounting for colon wall and peritoneal band in the zone where both tissues were superimposed (a stiffer zone corresponding to the induced stenosis).

In order to carry out the stent deployment an introducer sheath was placed inside the colon crossing the stenosis. An adequate catheter was used to place the stent at the exact deployment site, injecting contrast medium for checking the exact position. Once the stent was positioned, the stent was deployed by keeping it in place with the pusher and sliding the introducer sheath backwards. Fluoroscopic images of the stenosis were taken before and after deployment to verify the appropriate deployment [14]. Finally, endoscopic images were taken in different steps during the peristaltic movement.

\section{Results and Discussion}

The expansion process from the slotted tube leads to a bellshaped stent with diameters of $24.5 \mathrm{~mm}$ and $35.0 \mathrm{~mm}$ at the

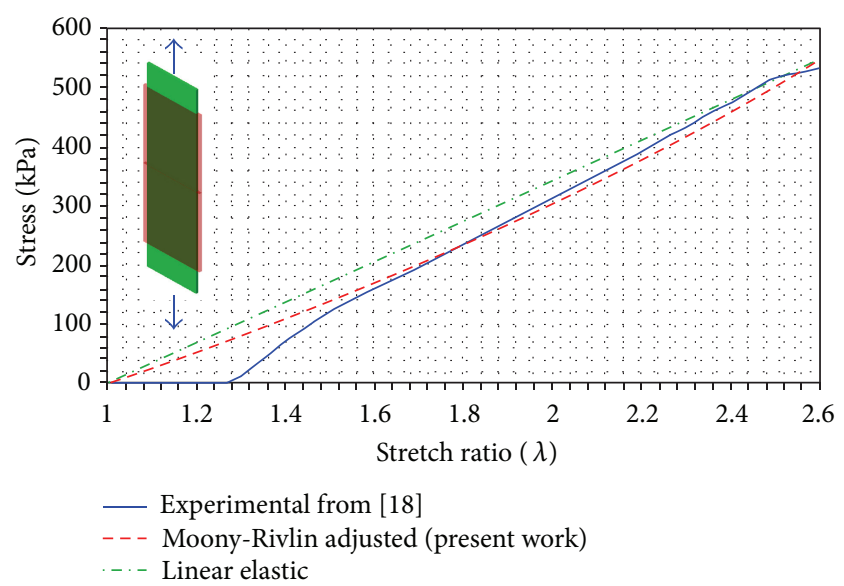

Figure 5: Adjustment of parameters for Mooney-Rivlin hyperelastic model of colonic tissue from the results reported in [18] for large bowel. $C_{10}=42.20 \mathrm{MPa} ; C_{01}=2.11 \mathrm{MPa} ; D_{1}=0$ (correlation coefficient $\left.R^{2}=0.993\right)$. Stretch ratio: actual length/initial length.

respective ends. Those values agree with the actual stent dimensions.

Stent introduction into a $14 \mathrm{Fr}$ catheter was carried out as depicted in Figure 6. The stent achieves a cylindrical form with an outer diameter corresponding to the inner diameter of the catheter. Phase transformation austenite-martensite was detected at the knot, but in any case, the maximum strain reaches the value of $\varepsilon_{L}$.

Concerning stent deployment, the process is shown in Figure 7. A perfect deployment can be observed with a final shape very close to the initial undeformed configuration and with diameters of $20.5 \mathrm{~mm}$ and $27.5 \mathrm{~mm}$ at the respective ends. So a diameter decrease of $16.3 \%$ and $21.4 \%$, respectively, appears as consequence of colon stiffness. The previous situation implies a compressive force exerted by the stent on the colon wall. Figure 8 shows the stresses in the colon wall due to that compressive force along the liberation process. It can be observed that as the stent is released from the catheter, a contact between stent and colon walls appears. In the final position, three zones of maximum compression are detected.

When the deployment starts, a first contact zone is detected at the colon proximal end. The deployment progress enables another contact zone correspondent to the medial curvature of the stent. Finally, when the complete deployment is reached, a contact zone appears at the colon distal end. Any case, the stresses in the colon wall never reach dangerous values capable of producing damage in the tissue.

Finally, for the peristaltic movement simulation, Figure 9 shows a comparison between FE deformed geometries and endoscopic images along the movement sequence. It can be observed how both sequences are coincident and how the stent recovers the shape previous to the peristaltic movement effect and remains in its original position without any displacement along colon, avoiding thus stent migration. 


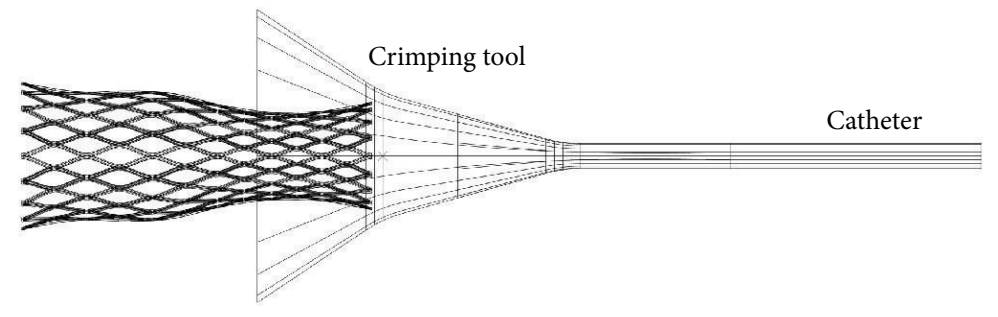

(a)

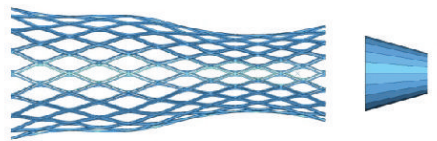

(1)

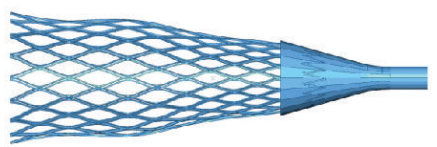

(2)

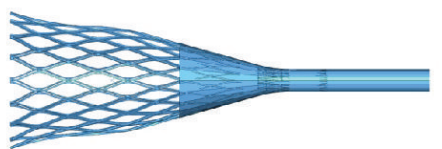

(3)

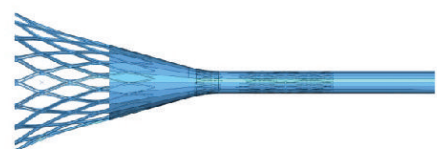

(4)

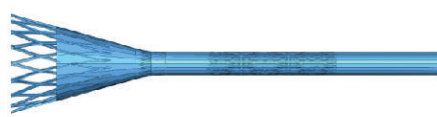

(5)

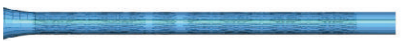

(6)

(b)

FIgURE 6: (a) Model of rigid surface used to crimp tool and catheter, (b) simulation of stent introduction into the catheter.

An essential topic concerns the strain and stress values reached in the stent with the maximum peristaltic compression, capable to cause the stent crushing. The strain and stress maps corresponding to the maximum peristaltic compression in the medial zone of the stent are shown in Figures 10 and 11, respectively. Similar maps can be depicted at any step of the peristaltic movement. All the strain values remain under $\varepsilon_{L}$, so no yielding occurs, and the stent is capable of recovering its initial shape and size, so crushing is not a problem.

In the clinical experimentation, the stent presented a good deployment with very good flexibility. The observed diameters corresponding to maximum expansion, measured on fluoroscopic images, were $19.5 \mathrm{~mm}$ and $25.5 \mathrm{~mm}$ at the respective ends $(-4.9 \%$ and $-7.3 \%$ with respect to the $\mathrm{FE}$ simulation results). The stent behavior during peristaltic movements was excellent, expanding without difficulty from the very narrow lumen corresponding to the maximum peristaltic pressure till the complete recovery of operative lumen when the pressure disappears (Figure 9).

Advantages of stenting in the colon obstruction before surgical resection include acute restoration of luminal patency with preoperative decompression, clinical stabilization and a proper colon cleansing, and the allowance of time for surgical optimization and staging before resection, so it is possible to perform the surgical intervention in a single stage. Complication rates associated with SEMS range between 14 and $42 \%$ [21-25], and most complications are minor.
Minor complications include SEMS occlusion due to tumor in growth; this may lead to recurrent colonic obstruction in $10-25 \%$ of endoscopically palliated cases [26]. Severe complications occur in up to $10 \%$ of patients. These consist of stent migration, perforation, and sepsis. Stent migration rates range between 10 and $12 \%$. Migration can happen a few days after stent deployment or later in the disease course. These were probably a result of tumor shrinkage from adjuvant chemotherapy [27] or to the effect of intestinal peristalsis. A complete study about stent migration and colon perforation can be found in [28].

While stents work well in the vascular structures, biliar track, or ureter, its operation is more difficult in the colon, because of the special peristalsis and fecal content characteristics, favoring its displacement or obstruction. The large intestine presents a complex peristalsis, consisting of deep circular and circular muscle contractions, causing a wave which progresses longitudinally along the intestine and leads to feces until the rectal ampoule.

Finite element simulation of NiTi colorectal stent has been carried out for a better understanding of the mechanical behaviour of a bell-shaped stent under peristaltic movements. It is a self-expanding stent with diamond cell type and bell-shaped profile with longitudinal variable radial strength getting a better resistance to the migration. The simulation results provide a good structural response to external pressure waves used to model the peristaltic movements. 

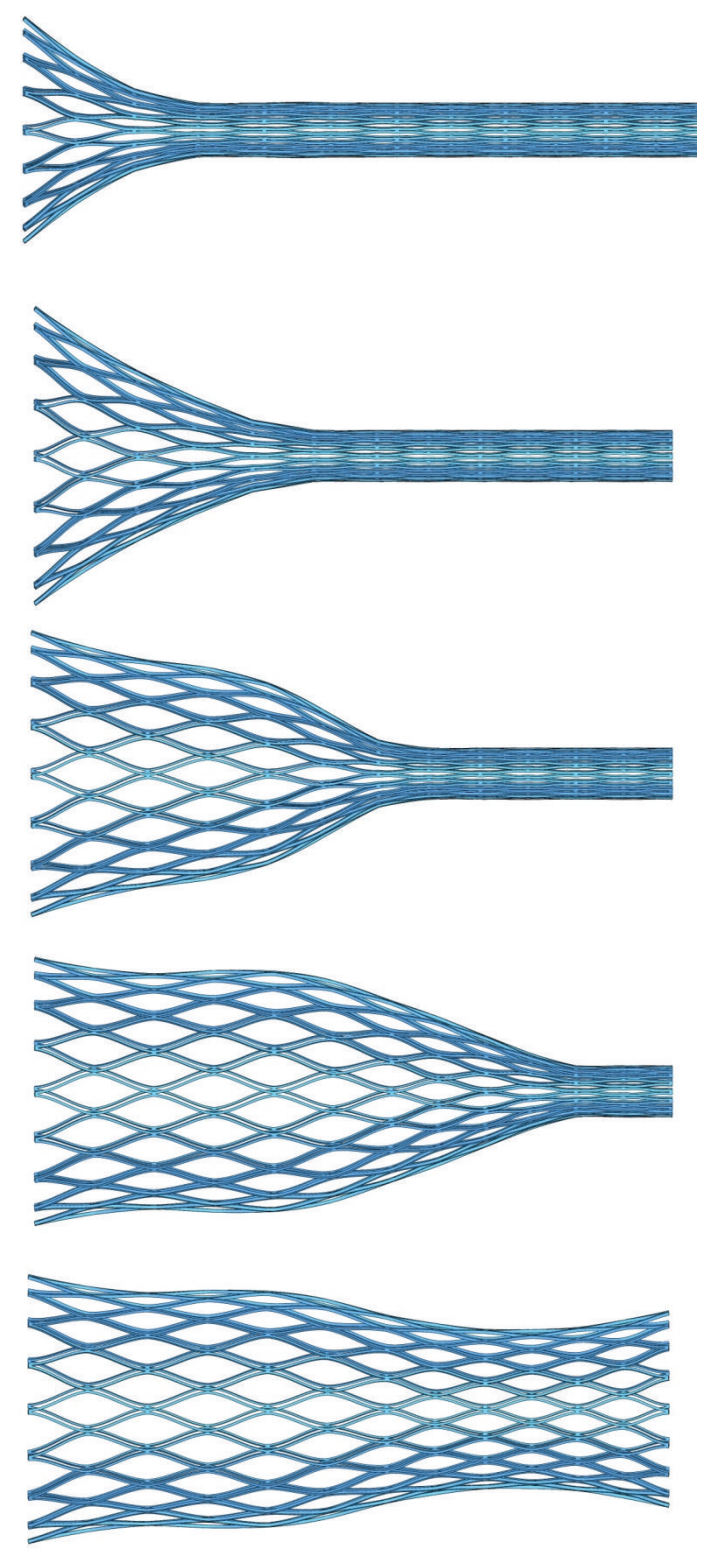

FIGURE 7: Simulation of stent deployment against the colon wall. Colon wall was removed to see the stent shape.

In validating colonic stent, we found that finite element analysis reproduces the stent compression-deployment process adequately by comparison with clinical experimentation. The idealized cylindrical straight geometry assumed to model colonic duct wall makes possible the application of peristaltic reflex and has a good approximation of the mechanical response of the stent structure. The modeled peristaltic pressure wave is in accordance with $[29,30]$. Clearly, patient-specific colonic duct exhibits wide variations in terms of geometry, size, material properties, and loading conditions. These simplifying assumptions somewhat limit the present results; nevertheless, the analysis introduces a new approach to evaluate the mechanical response of stents under peristaltic movements. The analyzed

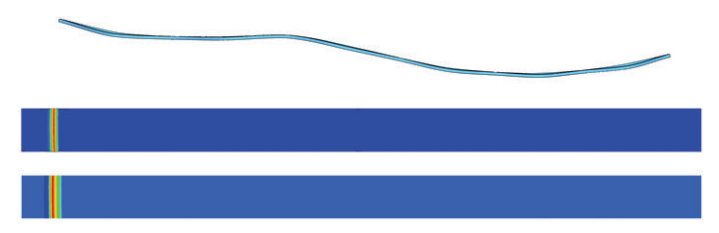

(a)

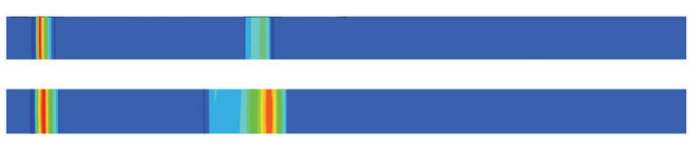

(b)

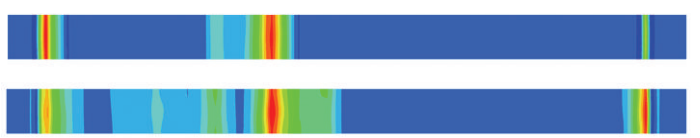

(c)

FIGURE 8: Stresses at the colon wall along deployment process, (a) Stress appearing and growing when distal end is released; (b) stress appearing and growing when intermediate bell-shaped zone is released; and (c) stress appearing and growing when proximal end is released.

stent has proved to be adequate to prevent migration and crushing.

The other simulated processes (expansion, introduction into the catheter and deployment) have demonstrated the ability of the analyzed stent for supporting every step in the clinical process required to the stent implantation, and hence the self-expanding stent with diamond cell type and bellshaped profile with longitudinal variable radial strength is a design reliable for colonic applications.

An essential point in the simulation is the approach used for the colonic tissue. In the specialized literature, several works about mechanical behaviour of soft tissue in coronary arteries [31, 32] or esophagus [33] can be found, but there are only a few studies concerning mechanical behaviour of colonic tissue $[18,20]$. For future improvements, in [18] experimental data for colonic tissue are included; those data could be used to adjust an accurate constitutive model.

Another important topic concerns fatigue behaviour of NiTi alloy. In this work, only one peristaltic pressure cycle has been simulated, but the peristaltic movements produce continuous cycling loads. Moreover, the cycling loads vary from zero to the maximum amplitude, corresponding to the maximum expansion rate in the stent. Cycling loads would cause stent breaks by cumulative damage and fatigue. However, the frequency of peristaltic movements is less than the corresponding to coronary stents [34].

NiTi alloys fatigue behaviour has been studied for applications with higher number of cycles than the corresponding to gastrointestinal ducts with good results [35] but with less cycle amplitudes. However other works are close to the present problem; so in [36], the approach of Coffin-Manson is applied to determine the fatigue life in NiTi medical devices, and in $[37,38]$, experimental results for NiTi low-cycle fatigue life are presented. In both cases (high-cycle, and low cycle 

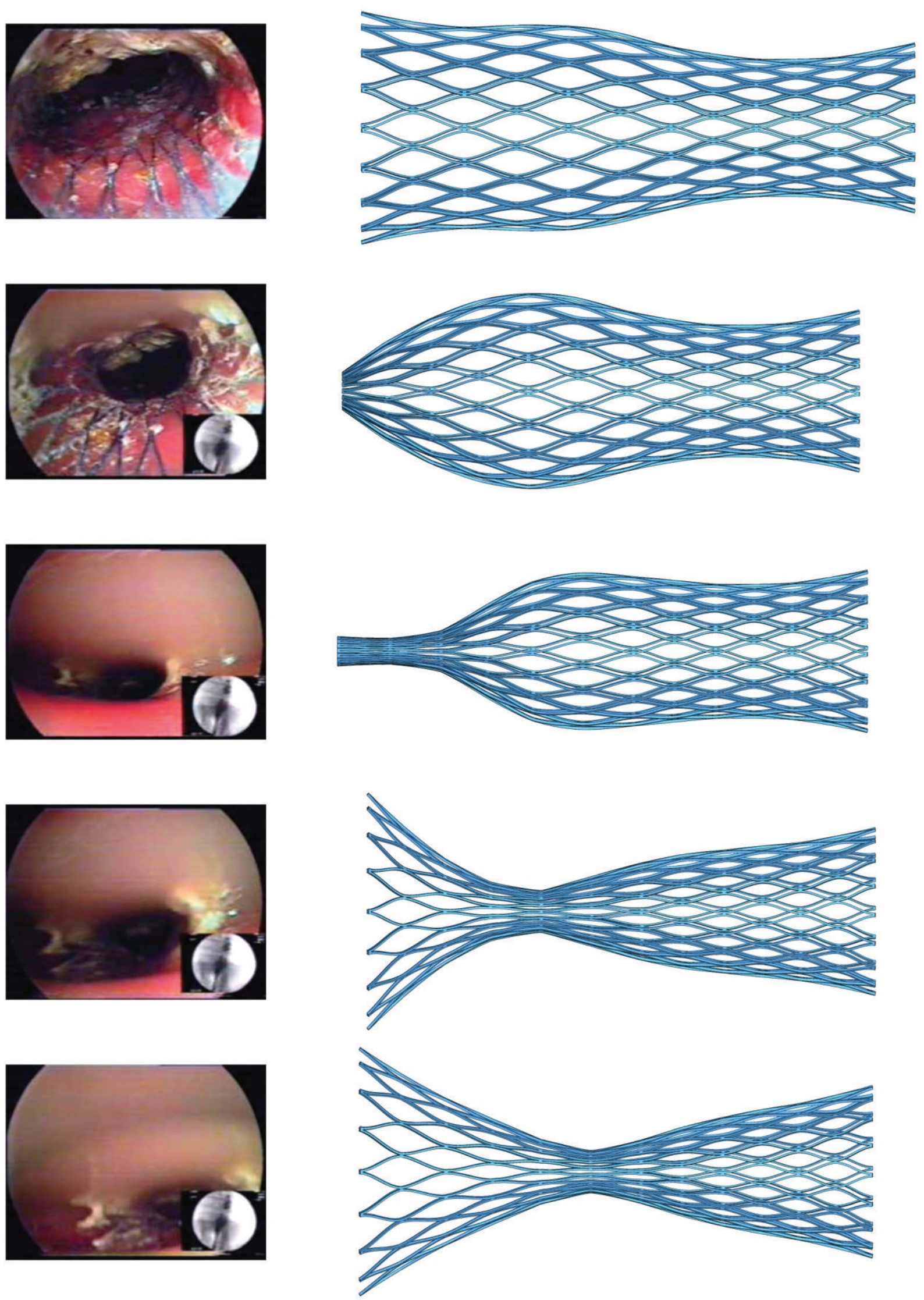

Figure 9: Continued. 

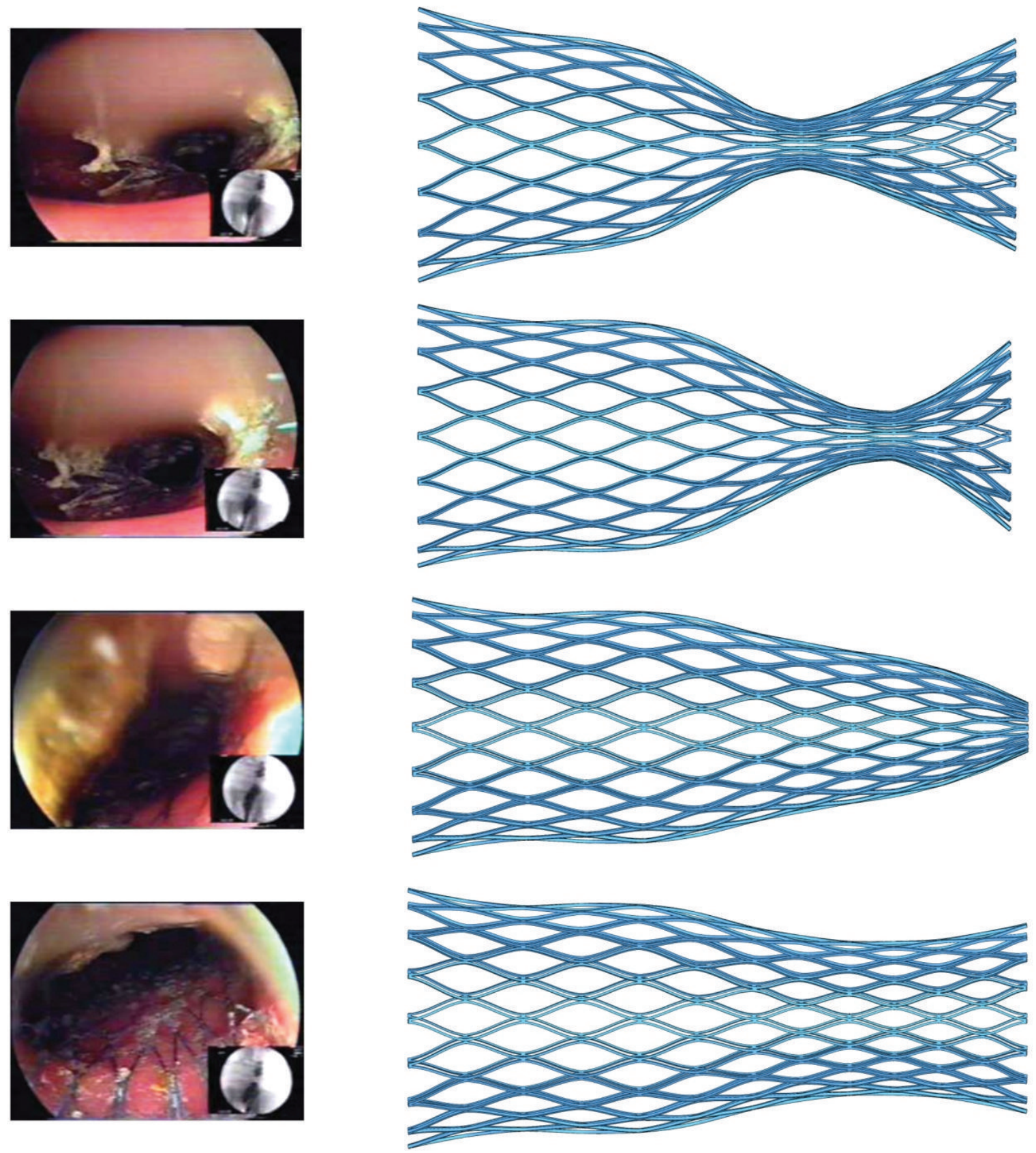

FIGURE 9: Peristaltic movement. Comparison between FE simulation and endoscopic images.

fatigue life), the ability of NiTi alloy to undergo cycling loads causing fatigue is demonstrated, so the stent would be able to undergo continuous cycling loads produced by the peristaltic movements.

\section{Conclusions}

A finite element simulation and a clinical experimentation of a bell-shaped nitinol stent functionality have been done. Every step in the implantation process was simulated (catheter introduction, releasing at position, and the effect of peristaltic wave). The stent presented a good deployment and flexibility, both in the simulation and in the clinical experimentation. The stent behavior during peristaltic movements was excellent, expanding without difficulty from the very narrow lumen corresponding to the maximum peristaltic pressure till the complete recovery of operative lumen when the pressure disappears.

The results of the study show that Niti self-expanding bellshaped stent is as an effective alternative to relieve colonic 


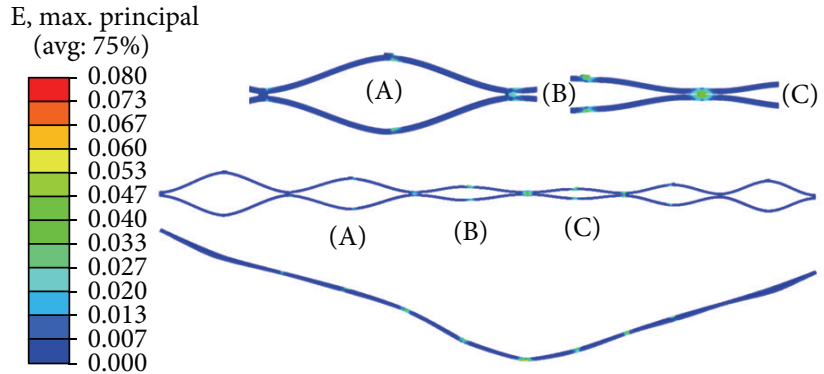

Figure 10: Strain distribution corresponding to the maximum peristaltic compression in the medial zone.

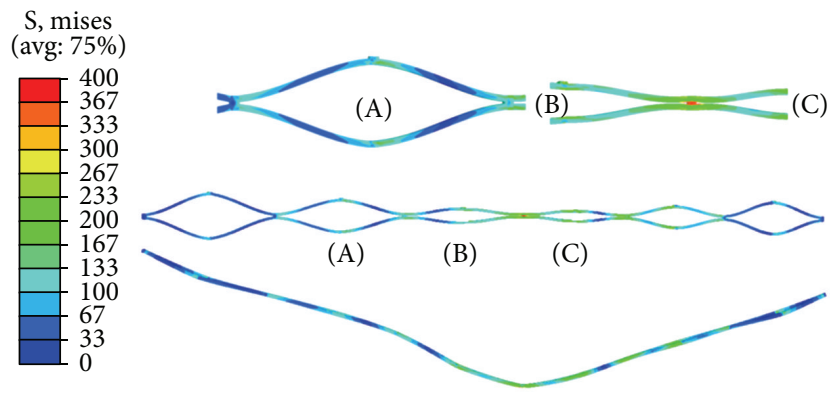

FIGURE 11: von Mises stress distribution corresponding to the maximum peristaltic compression in the medial zone.

obstruction, either as a bridge to elective single-stage surgery avoiding a stoma or as a definitive palliative solution in patients with irresectable tumor or poor estimated survival.

\section{Conflict of Interests}

The authors declare that they have no conflict of interests.

\section{Authors' Contributions}

J. A. Puértolas conceived the original stent design and manufacturing process. E. Bajador and A. Herrera conceived and developed the clinical aspects of the work. S. Puértolas, E. López, E. Ibarz, and L. Gracia conceived and developed the finite element model and carried out all the simulations. L. Gracia and A. Herrera coordinated the work between surgeons and engineers. All authors participated in the drawing up of the paper, and read and approved the final version.

\section{Acknowledgment}

The authors gratefully acknowledge "Jesús Usón" Minimally Invasive Surgery Center (Cáceres, Spain) for planning and execution of clinical experimentation.

\section{References}

[1] D. M. Parkin, F. Bray, J. Ferlay, and P. Pisani, "Global cancer statistics, 2002," Ca-A Cancer Journal for Clinicians, vol. 55, no. 2, pp. 74-108, 2005.
[2] G. T. Deans, Z. H. Krukowski, and S. T. Irwin, "Malignant obstruction of the left colon," British Journal of Surgery, vol. 81, no. 9, pp. 1270-1276, 1994.

[3] I. M. Leitman, J. D. Sullivan, D. Brams, and J. J. DeCosse, "Multivariate analysis of morbidity and mortality from the initial surgical management of obstructing carcinoma of the colon," Surgery Gynecology and Obstetrics, vol. 174, no. 6, pp. 513-518, 1992.

[4] M. Dohmoto, "New method: endoscopic implantation of rectal stent in palliative treatment of malignant stenosis," Endoscopia Digestiva, vol. 3, pp. 1507-1512, 1991.

[5] S. Meisner, F. González-Huix, J. Vandervoort et al., "Selfexpanding metal stenting for palliation of patients with malignant colonic obstruction: effectiveness and efficacy on 255 patients with 12-month's follow-up," Gastroenterology Research and Practice, vol. 2012, Article ID 296347, 6 pages, 2012.

[6] S. Varadarajulu, A. Roy, T. Lopes, E. R. Drelichman, and M. Kim, "Endoscopic stenting versus surgical colostomy for the management of malignant colonic obstruction: comparison of hospital costs and clinical outcomes," Surgical Endoscopy and Other Interventional Techniques, vol. 25, no. 7, pp. 2203-2209, 2011.

[7] U. P. Khot, A. Wenk Lang, K. Murali, and M. C. Parker, "Systematic review of the efficacy and safety of colorectal stents," British Journal of Surgery, vol. 89, no. 9, pp. 1096-1102, 2002.

[8] S. Sebastian, S. Johnston, T. Geoghegan, W. Torreggiani, and M. Buckley, "Pooled analysis of the efficacy and safety of selfexpanding metal stenting in malignant colorectal obstruction," American Journal of Gastroenterology, vol. 99, no. 10, pp. 20512057, 2004.

[9] A. M. Watt, I. G. Faragher, T. T. Griffin, N. A. Rieger, and G. J. Maddern, "Self-expanding metallic stents for relieving malignant colorectal obstruction: a systematic review," Annals of Surgery, vol. 246, no. 1, pp. 24-30, 2007.

[10] J. Jiménez-Pérez, J. Casellas, J. García-Cano et al., "Colonic stenting as a bridge to surgery in malignant large-bowel obstruction: a report from two large multinational registries," American Journal of Gastroenterology, vol. 106, no. 12, pp. 21742180, 2011.

[11] S. Domingo, S. Puértolas, L. Gracia, and J. A. Puértolas, "Mechanical comparative analysis of stents for colorectal obstruction," Minimally Invasive Therapy, vol. 16, no. 2, pp. 126136, 2007.

[12] F. Etave, G. Finet, M. Boivin, J. Boyer, G. Rioufol, and G. Thollet, "Mechanical properties of coronary stents determined by using finite element analysis," Journal of Biomechanics, vol. 34, no. 8, pp. 1065-1075, 2001.

[13] J. Tambaca, S. Canic, M. Kosor, R. D. Fish, and D. Paniagua, "Mechanical behavior of fully expanded commercially available endovascular coronary stents," Texas Heart Institute Journal, vol. 38, no. 5, pp. 491-501, 2011.

[14] S. Domingo, S. Puértolas, L. Gracia-Villa, M. Mainar, J. Usón, and J. A. Puértolas, "Design, manufacture and evaluation of a NiTi stent for colon obstruction," Bio-Medical Materials and Engineering, vol. 15, no. 5, pp. 357-365, 2005.

[15] SIEMENS, http://www.plm.automation.siemens.com/

[16] F. Auricchio, "A robust integration-algorithm for a finite-strain shape-memory-alloy superelastic model," International Journal of Plasticity, vol. 17, no. 7, pp. 971-990, 2001.

[17] Dassault Système, http://www.3ds.com/ . 
[18] V. I. Egorov, I. V. Schastlivtsev, E. V. Prut, A. O. Baranov, and R. A. Turusov, "Mechanical properties of the human gastrointestinal tract," Journal of Biomechanics, vol. 35, no. 10, pp. 1417-1425, 2002.

[19] C. Lally and P. J. Prendergast, "An investigation into the applicability of a Mooney-Rivlin constitutive equation for modelling vascular tissue in cardiovascular stenting procedures," in Proceedings of the International Congress on Computational Biomechanics, pp. 542-550, 2003.

[20] H. Gregersen, Biomechanics of the Gastrointestinal Tract. New Prospectives in Motility Research and Diagnostics, Springer, London, UK, 2002.

[21] A. Mainar, M. A. De Gregrio Ariza, E. Tejero et al., "Acute colorectal obstruction: treatment with self-expandable metallic stents before scheduled surgery-results of a multicenter study," Radiology, vol. 210, no. 1, pp. 65-69, 1999.

[22] S. Athreya, J. Moss, G. Urquhart, R. Edwards, A. Downie, and F. W. Poon, "Colorectal stenting for colonic obstruction: the indications, complications, effectiveness and outcome-5-Year review," European Journal of Radiology, vol. 60, no. 1, pp. 91-94, 2006.

[23] S. H. Baik, N. K. Kim, H. W. Cho et al., "Clinical outcomes of metallic stent insertion for obstructive colorectal cancer," Hepato-Gastroenterology, vol. 53, no. 68, pp. 183-187, 2006.

[24] P. W. G. Carne, J. N. R. Frye, G. M. Robertson, and F. A. Frizelle, "Stents or open operation for palliation of colorectal cancer: a retrospective, cohort study of perioperative outcome and longterm survival," Diseases of the Colon and Rectum, vol. 47, no. 9, pp. 1455-1461, 2004.

[25] A. J. M. Watson, V. Shanmugam, I. Mackay et al., "Outcomes after placement of colorectal stents," Colorectal Disease, vol. 7, no. 1, pp. 70-73, 2005.

[26] C. A. Ely and M. E. Arregui, "The use of enteral stents in colonic and gastric outlet obstruction," Surgical Endoscopy and Other Interventional Techniques, vol. 17, no. 1, pp. 89-94, 2003.

[27] F. Camuñez, A. Echenagusia, G. Simo, F. Turégano, J. Vázquez, and I. Barreiro-Meiro, "Malignant colorectal obstruction treated by means of self-expanding metallic stents: effectiveness before surgery and in palliation," Radiology, vol. 216, no. 2, pp. 492-497, 2000.

[28] H. K. Jin, H. Song, Y. Li et al., "Dual-design expandable colorectal stent for malignant colorectal obstruction: comparison of flared ends and bent ends," American Journal of Roentgenology, vol. 193, no. 1, pp. 248-254, 2009.

[29] M. Mishra and A. R. Rao, "Peristaltic transport in a channel with a porous peripheral layer: model of a flow in gastrointestinal tract," Journal of Biomechanics, vol. 38, no. 4, pp. 779-789, 2005.

[30] S. K. Pandey, M. K. Chaube, and D. Tripathi, "Peristaltic transport of multilayered power-law fluids with distinct viscosities: a mathematical model for intestinal flows," Journal of Theoretical Biology, vol. 278, no. 1, pp. 11-19, 2011.

[31] C. Lally, F. Dolan, and P. J. Prendergast, "Cardiovascular stent design and vessel stresses: a finite element analysis," Journal of Biomechanics, vol. 38, no. 8, pp. 1574-1581, 2005.

[32] G. A. Holzapfel, "Determination of material models for arterial walls from uniaxial extension tests and histological structure," Journal of Theoretical Biology, vol. 238, no. 2, pp. 290-302, 2006.

[33] A. N. Natali, E. L. Carniel, and H. Gregersen, "Biomechanical behaviour of oesophageal tissues: material and structural configuration, experimental data and constitutive analysis," Medical Engineering and Physics, vol. 31, no. 9, pp. 1056-1062, 2009.
[34] L. Miller, Q. Dai, A. Korimilli, B. Levitt, Z. Ramzan, and J. Brasseur, "Use of endoluminal ultrasound to evaluate gastrointestinal motility," Digestive Diseases, vol. 24, no. 3-4, pp. 319-341, 2006.

[35] A. R. Pelton, V. Schroeder, M. R. Mitchell, X. Y. Gong, M. Barney, and S. W. Robertson, "Fatigue and durability of Nitinol stents," Journal of the Mechanical Behavior of Biomedical Materials, vol. 1, no. 2, pp. 153-164, 2008.

[36] A. Runciman, D. Xu, A. R. Pelton, and R. O. Ritchie, "An equivalent strain/Coffin-Manson approach to multiaxial fatigue and life prediction in superelastic Nitinol medical devices," Biomaterials, vol. 32, no. 22, pp. 4987-4993, 2011.

[37] M. G. de Azevedo Bahia, R. Fonseca Dias, and V. T. L. Buono, "The influence of high amplitude cyclic straining on the behaviour of superelastic NiTi," International Journal of Fatigue, vol. 28, no. 9, pp. 1087-1091, 2006.

[38] A. M. Figueiredo, P. Modenesi, and V. Buono, "Low-cycle fatigue life of superelastic NiTi wires," International Journal of Fatigue, vol. 31, no. 4, pp. 751-758, 2009. 

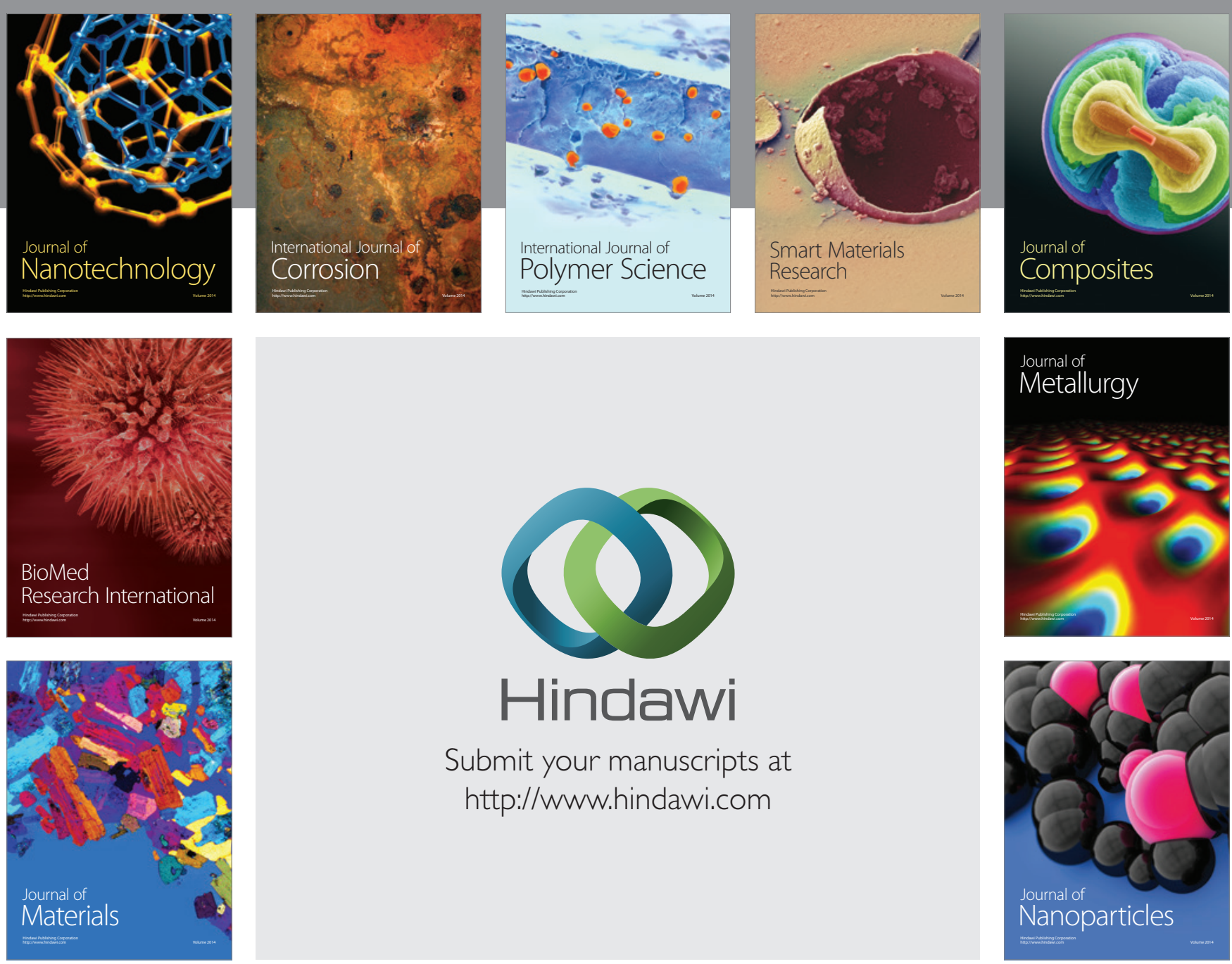

Submit your manuscripts at http://www.hindawi.com
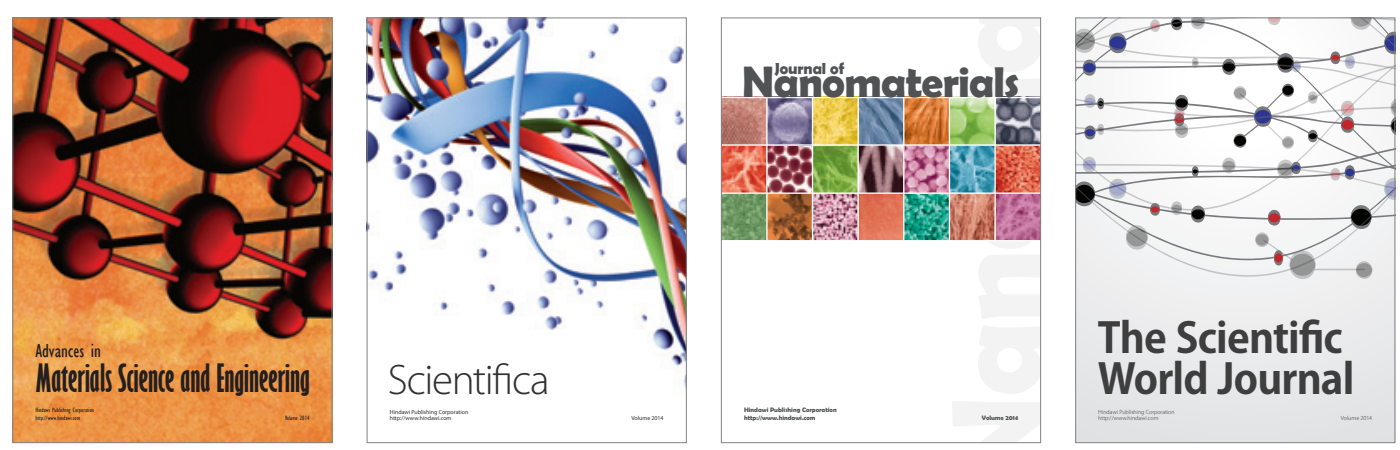

\section{The Scientific World Journal}
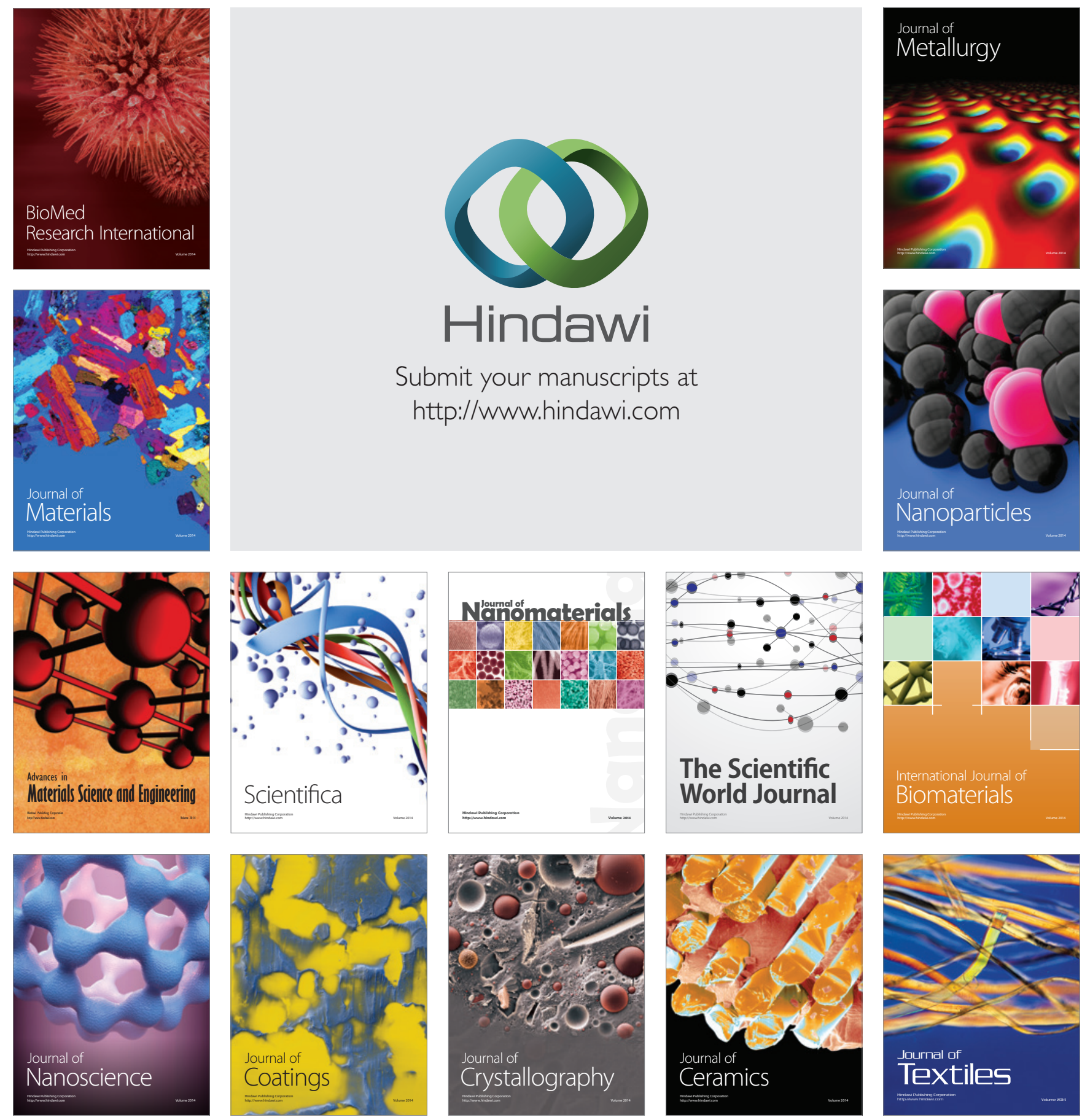